\title{
A jigsaw puzzle metamaterial concept ${ }^{\text {th }}$
}

\author{
V. Nežerka ${ }^{\mathrm{a}}$, M. Somr ${ }^{\mathrm{a}}$, T. Janda ${ }^{\mathrm{a}}$, J. Vorel ${ }^{\mathrm{a}}$, M. Doškár̆a ${ }^{\mathrm{a}}$ J. Antoš ${ }^{\mathrm{a}}$, J. Zeman $^{\mathrm{a}}$, J. Novák ${ }^{\mathrm{a}, *}$ \\ ${ }^{a}$ Faculty of Civil Engineering, Czech Technical University in Prague, Thákurova 7, 16629 Praha 6, Czech Republic
}

\begin{abstract}
A concept of a planar modular mechanical metamaterial inspired by the principle of local adaptivity is proposed. The metamaterial consists of identical pieces similar to jigsaw puzzle tiles. Their rotation within assembly provides a substantial flexibility in terms of structural behavior, whereas mechanical interlocks enable reassembly. The tile design with a diagonal elliptical opening allows us to vary elastic properties from stiff to compliant, with positive, zero, or negative Poisson's ratio. The outcomes of experimental testing on additively manufactured specimens confirm that the assembly properties can be accurately designed using optimization approaches with finite element analysis at heart.
\end{abstract}

Keywords: modular metamaterial, auxetic behavior, additive manufacturing, customized assembly, digital image correlation

\section{Introduction}

Nature efficiently distributes material and designs optimal structure across scales with respect to anticipated loading. Bone tissue is a perfect example of such a multiscale hierarchical structure in which the organization of mineral nanoparticles and collagen microfibrils governs the bone's elastic properties [1]. Here we propose to mimic such local adaptivity by material design similarly to a way children assemble images decomposed into jigsaw puzzle pieces. We create a planar block of mechanical metamaterial [2] with required overall elastic stiffness emanating from the geometry and composition of mesostructural units, similar to the concepts proposed in, e.g., [3-6]. In particular, the assemblies are composed of mutually rotated identical tiles with elliptic openings, Figure 1, as proposed by Taylor et al. [7]. In addition, the subscale, tile-level, geometry design also allows us to tune Poisson's ratio $[9,10]$ and thus fabricate materials exhibiting conventional or auxetic $[11,12]$ behavior. The tiles in assemblies can be connected through jigsaw puzzle interlocks [13], or the modularity concept can serve for optimization and design purposes and the conglomerates can be printed out as monolithic pieces [4]. In this work, the interlocks are not glued together, they utilize only frictional forces to meet the requirement on reasonably stiff contact and non-destructive reassembly. In order to obtain comprehensive full-field information on strain and

\footnotetext{
Author's post-print version of the article manuscript published in Composite Structures DOI: 10.1016/j.compstruct.2018.06.015.

* Corresponding author

Email address: novakja@fsv. cvut. cz (J. Novák)
}

(C) 2018. This manuscript version is made available under the CC-BY-NC-ND 4.0 license. 


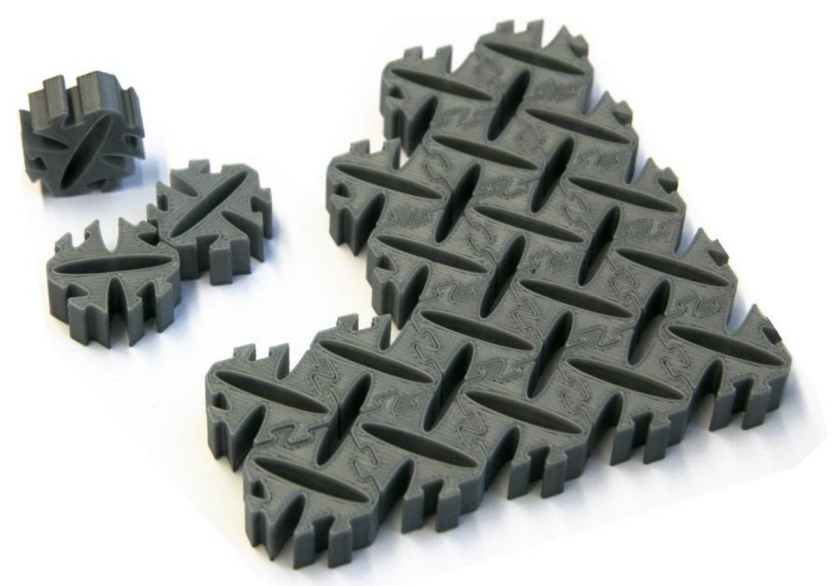

Figure 1: Additively manufactured jigsaw puzzle tiles and partly disassembled auxetic aggregate with effective Poisson's ratio equal to -0.55 .

displacement fields, a digital image correlation (DIC) $[15,16]$ analysis is employed throughout the study.

\section{Modular concept}

The main ingredient of the proposed concept are square tiles of a jigsaw puzzle-like shape, as shown in Figure 2(a). Besides the obvious assembly into periodic arrangements, Figure 2(b), the rotational symmetry of the interlocks allows us to perturb the regular arrangement leading to locally adjusted elastic behavior [14].

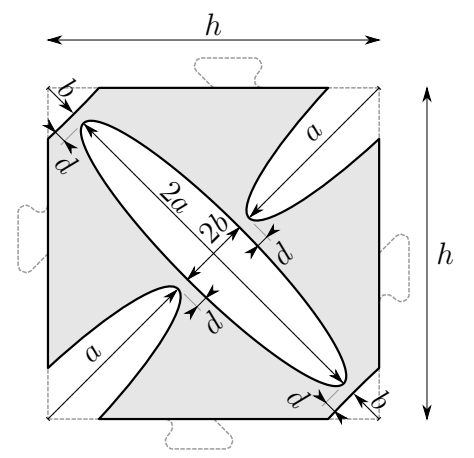

(a)

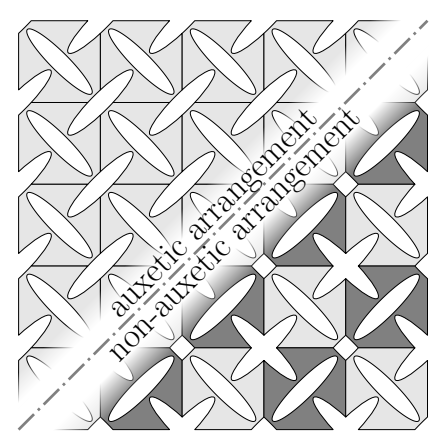

(b)

Figure 2: (a) geometry of single jigsaw puzzle tile with interlocks outlined by dotted contours. (b) regular assemblies into either auxetic or non-auxetic arrangements. Highlighted tiles in the latter arrangement are rotated by $90^{\circ}$. Particular dimensions of individual parameters of single tile are derived from the tile edge length $h$, here set to $20 \mathrm{~mm}$, as follows: major semiaxis $a=h / 2$, minor semiaxis $b=h / 10$, yielding ligament thickness $d \approx h / 12$. 


\section{Metamaterial assembly}

To illustrate the adaptivity through local tile rotations, assemblies consisting of $5 \times 5$ tiles were arranged to exhibit auxetic, non-auxetic, and mixed behavior and subjected to a displacementcontrolled compression applied at the top edge/facet of the specimen. First, we explored two

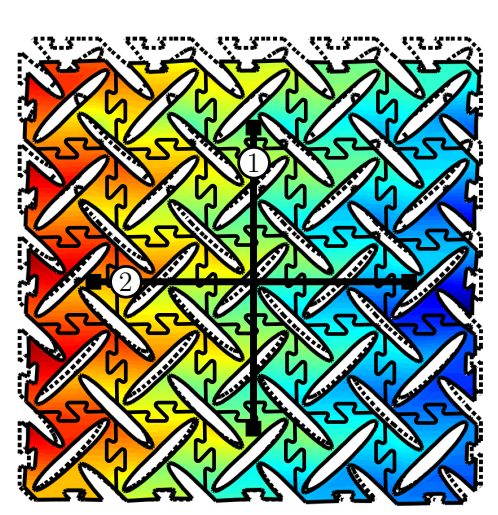

(a)

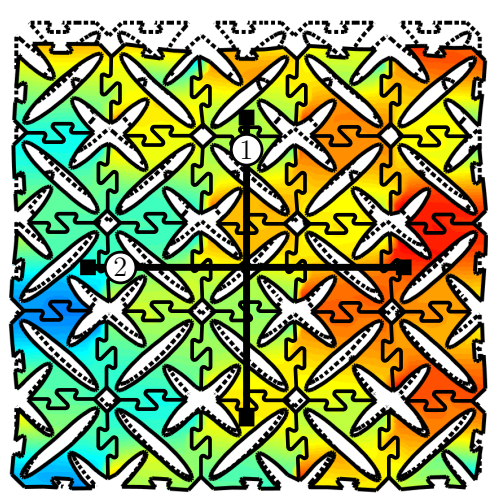

(b)

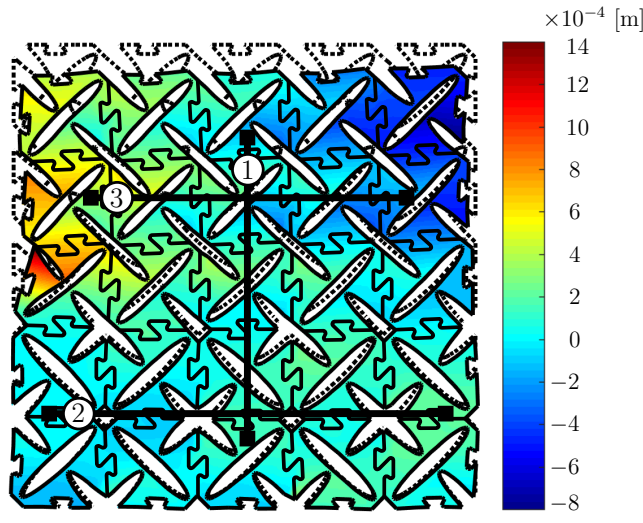

(c)

Figure 3: Specimen deformation (original shape indicated by dotted contours) and horizontal displacement distribution maps, obtained by DIC, under uniaxial compression applied to (a) auxetic, (b) non-auxetic, and (c) mixed assemblies. Virtual extensometers are indicated with boldface lines. Positive displacements are measured according to $x$ and $y$ axes pointing right- and up-wards from the specimen center.

regular cases: (i) an auxetic arrangement and (ii) a non-auxetic arrangement, see Figures 2(b) and $3(a, b)$. In addition to the two configurations illustrated also in Figure 4(a,b), a mixed arrangement (iii) supposed to combine the behavior of previous assemblies was also experimentally tested, Figures 3(c) and 4(c).

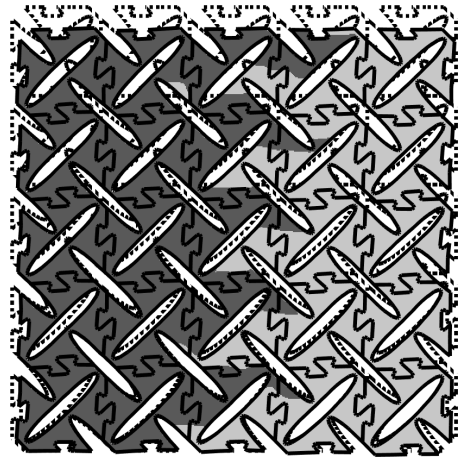

(a)

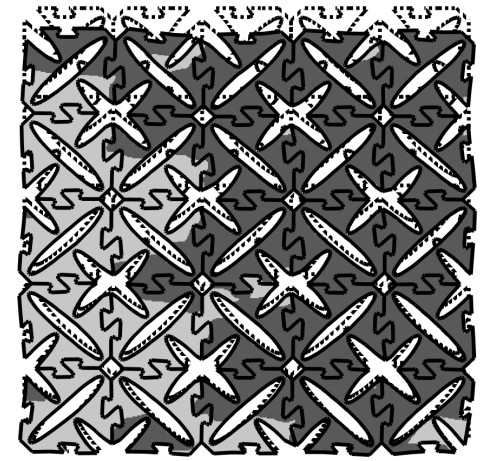

(b)

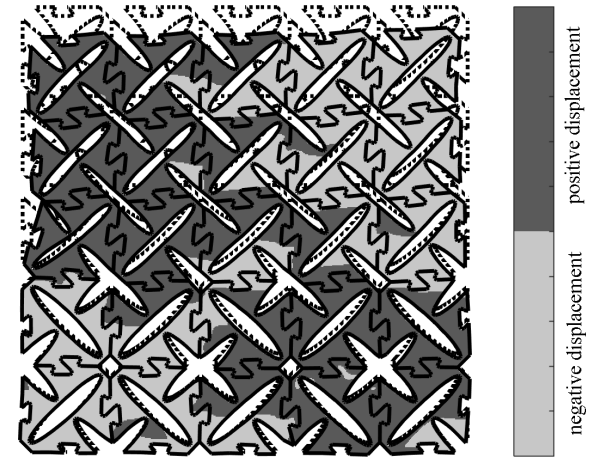

(c)

Figure 4: Specimen deformations with binary representation of horizontal displacements, obtained by DIC, under uniaxial compression applied to (a) auxetic, (b) non-auxetic, and (c) mixed assemblies. Positive displacements are measured according to $x$ and $y$ axes pointing right- and up-wards from the specimen center.

The regular zigzag arrangement of ellipses (i) yielded the smallest value for both elastic constants, Young's modulus and Poisson's ratio. As expected, the specimen contracted in horizontal 
direction when compressed vertically, Figure 5(b), demonstrating the desired auxetic response. On the other hand, the configuration (ii) with star-like openings exhibited twice as stiff response, see Figure 5(a), and Poisson's ratio exceeding the one of the virgin polylactic acid (PLA) filament. The deformation of the mixed arrangement (iii) clearly proves that Poisson's ratio can be tuned locally, the bottom layer expands horizontally, while the upper part exhibits the auxetic behavior, Figure 5(b). This is also evident from the binary patterns in Figure 4(c).

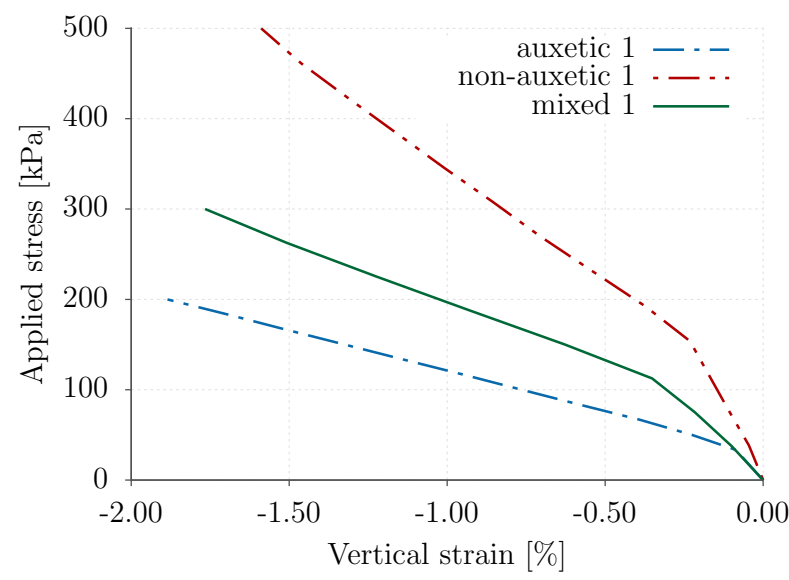

(a)

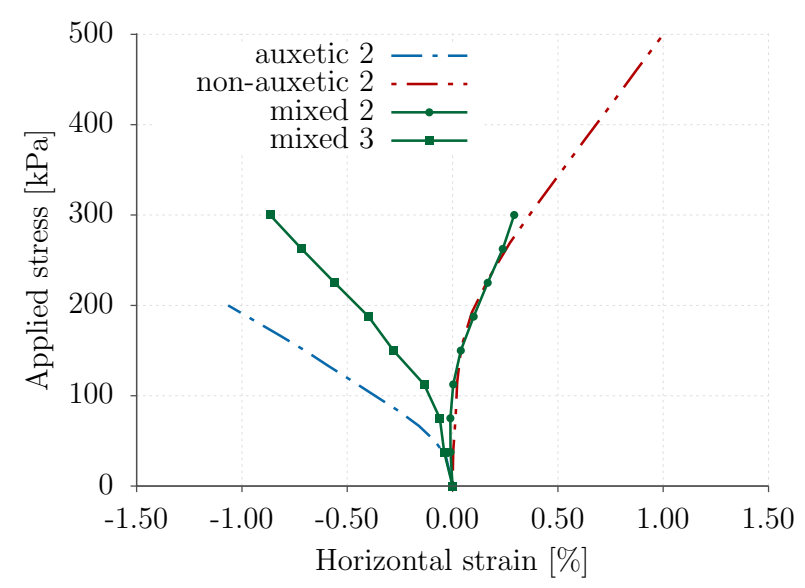

(b)

Figure 5: Effective stress-strain curves for (a) vertical and (b) horizontal extensometers placed in auxetic, non-auxetic, and mixed assemblies according to Figure 3.

\section{Customized assemblies}

The merit of the proposed material system goes beyond many periodic metamaterial designs [1719] because of its modularity [3, 5, 20] and inherent aperiodicity [14]. Rotation of a few tiles by $90^{\circ}$ within an assembly significantly changes the response to loading. The assembly plan can be adjusted with respect to anticipated loading and specific requirements on both local and global behavior [21, 22]. To demonstrate this feature, two modular assemblies (fabricated without interfaces and with imperfect mechanical interlocks) of $4 \times 4$ tiles were optimized employing a finite element analysis to eliminate tilt angle $\phi$ due to eccentric loading, as indicated in Figure 6(a).

Two variants of the customized assemblies were considered. First, interfaces between individual tiles were considered perfectly rigid as these are way easier to handle within the numerical framework. Tested assemblies were then manufactured as monoliths, but with the modular design strategy with compatible meshes without contacts in mind. Second, imperfect contacts within aggregates with mechanical interlocks were introduced into the finite element model by means of contact elements and the entire procedure from design to fabrication sustained fully modular.

The tilt angle $\phi$ of the upper edge across all admissible assembly combinations ranged from $-0.100^{\circ}$ to $1.912^{\circ}$ for solid assemblies and from $-0.108^{\circ}$ to $2.249^{\circ}$ for those with imperfect contacts. The optimal assemblies that were supposed to yield zero tilt angle, both solid and with mechanical interlocks, were additively manufactured and tested to validate the numerical model. 
As expected, the optimum assembly plan with mechanical interlocks was completely different from the one with fixed contacts, as shown in Figure 6(b,c), demonstrating the key role of connections. The experimental tilt angle was measured from the displacement distribution maps using virtual extensometers within the DIC framework. The optimum solid and mechanically connected assemblies yielded $\phi=-0.00022^{\circ}$ and $\phi=0.01^{\circ}$, respectively.

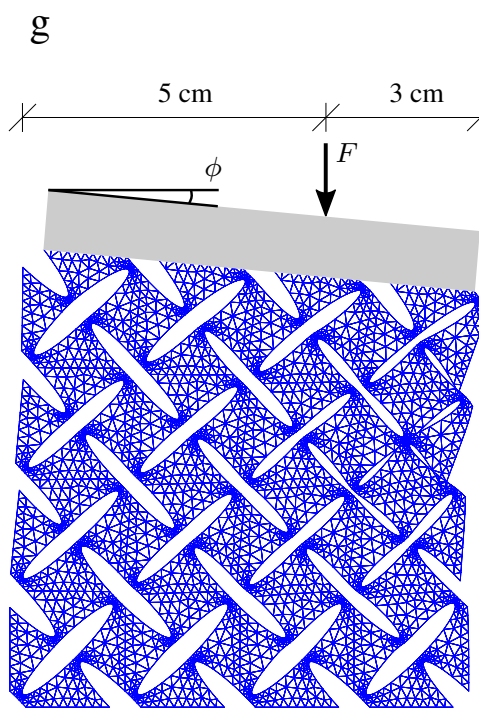

(a)

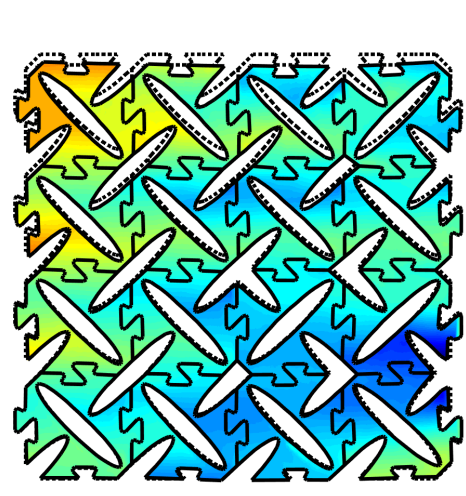

(b)

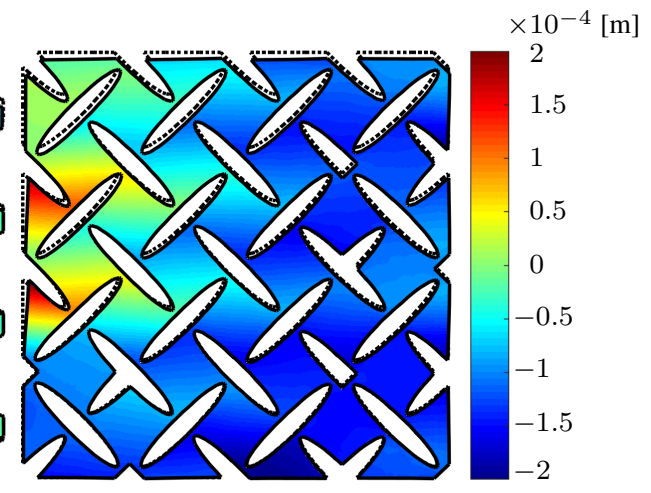

(c)

Figure 6: (a) eccentric loading scheme and the measured tilt angle $\phi$. Experimentally obtained horizontal displacements (original shape indicated by dotted contours) obtained by DIC measurements on specimens designed to compensate eccentric loading: (b) assembly with imperfect mechanical interlocks and (c) without interfaces.

\section{Materials and methods}

\subsection{Optimization of customized assemblies.}

In the spirit of the explicit complete enumeration method, the computation of all $2^{16}=65,403$ combinations of $4 \times 4$ assemblies with the selection of the best solution was done using in-house scripts developed in MATLAB ${ }^{1}$ software linked to ANSYS $^{2}$. These were discretized by 6,184 linear triangular (assemblies without interfaces, see Figure 6(a)) and 13, 322 isoparametric fournode quadrilateral (assemblies with imperfect interlocks) finite elements PLANE182 under plane strain assumptions. Properties of the contact elements, used for simulations of imperfect interfaces between tiles, were calibrated based on experimental measurements and inverse calculations on pairs of interlocked tiles. In particular, the augmented Lagrangian contact algorithm was employed with the automatic update, using the default values for interfacial contact stiffness parameters provided by the ANSYS program.

\footnotetext{
${ }^{1}$ The MathWorks, Inc., Matlab release 2015a, http: / / www . mathworks. com/products/mat lab/, Natick, Massachusetts, U.S.

${ }^{2}$ ANSYS Mechanical, Academic research, release 16.2, http: / /www . ansys . com/, Pennsylvania, U.S.
} 


\subsection{Fabrication and materials.}

The $2 \times 2 \times 1 \mathrm{~cm}$ tiles for experimental verification were additively manufactured from PLA filament with Young's modulus of about 1, $800 \mathrm{MPa}$ and Poisson's ratio equal to 0.3 [23], using Prusa i3 3D printer.

\subsection{Mechanical testing and data acquisition.}

The aim of the experimental analysis was to measure macroscopic response of the tested assemblies to displacement-controlled compression. The experiments were carried out using LabTest 4.100SP1 testing machine. The $5 \times 5$ assemblies were loaded at rate $1.2-2.0 \mathrm{~mm} / \mathrm{min}$ until the displacement reached $1.6 \mathrm{~mm}$, while the optimized $4 \times 4$ assemblies were loaded at the same rate until the eccentrically applied force (see Figure 6) reached 200 N. In both cases, a steel plate of $1 \mathrm{~cm}$ in thickness was used to distribute the loading over the upper edge of the assemblies.

The analyzed images were taken in 2-second intervals by a high-definition camera Canon EOS 70D in uncompressed format (.raw), yielding a resolution of $12 \mathrm{px} / \mathrm{mm}$. The effect of lens distortion was minimized by setting the focal length to $55 \mathrm{~mm}$. A non-commercial open-source software Ncorr [16] was used to evaluate the fields, and a postprocessing of the DIC data was accomplished using an in-house software Ncorr_post ${ }^{3}$. Virtual extensometers were placed at distinct locations, as depicted in Figure 3, in order to quantify the relative vertical and horizontal displacements of the entire assemblies.

\section{Conclusions}

Presented results demonstrate that even such an elementary modular system based on a single unit provides a substantial flexibility in controlling overall mechanical properties by local rotations of mesostructural units by $90^{\circ}$. The outcomes of experimental tests performed on the additively manufactured specimens confirm that the assembly properties can be accurately designed using a numerical analysis and optimization. On the other hand, avoiding numerical analyzes in the

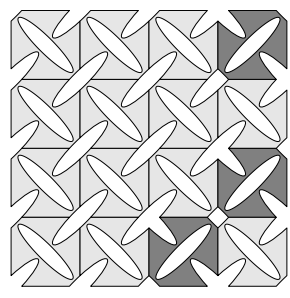

(a)

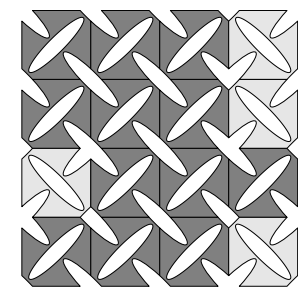

(b)

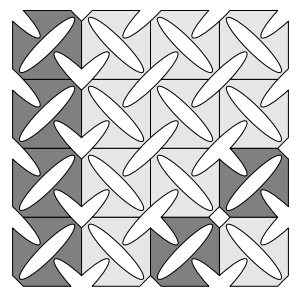

(c)

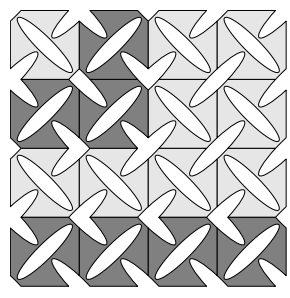

(d)

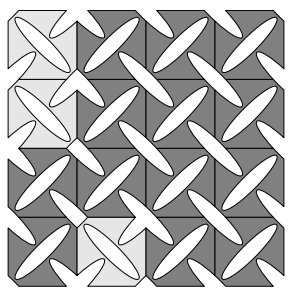

(e)

Figure 7: Solid assemblies for tilt angles (a) $\phi=-0.100^{\circ}$, (b) $\phi=0.273^{\circ}$, (c) $\phi=0.575^{\circ}$, (d) $\phi=0.912^{\circ}$, (e) $\phi=1.912^{\circ}$. Highlighted tiles are rotated by $90^{\circ}$ with respect to the setting adopted in Figure 2(a).

design process is difficult, given the emergence of highly-localized instability-like behavior when

\footnotetext{
${ }^{3}$ V. Nežerka, Ncorr_post v2.0: DIC Post-Processing Tool, http://mech.fsv.cvut.cz/ nezerka/DIC/ index.htm, Czech Technical University in Prague.
} 
snapping from one configuration to another (pronounced namely for aggregates with imperfect contacts). Because of this phenomenon, we were unable to devise any simple heuristic, analytical, or semi-analytical tool for predicting the overall assembly response a priori. To further highlight this difficulty, Figure 7 shows the intricate, often counter-intuitive dependence of the tilt angle on the tile configurations, whereas Figures 8 depicts two distinct assemblies delivering nearly the same structural response.

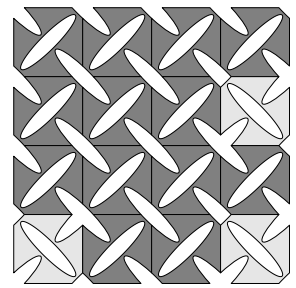

(a)

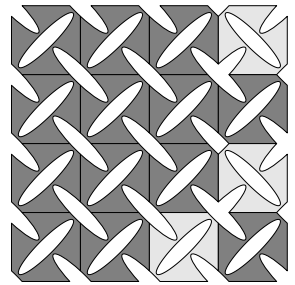

(b)

Figure 8: Solid assemblies for tilt angles, (a) $\phi=0.00022^{\circ}$, (b) $\phi=0.00029^{\circ}$. Highlighted tiles are rotated by $90^{\circ}$ with respect to the setting adopted in Figure 2(a).

Nonetheless, to the merit of the proposed modular concept, it is scalable up to the limits of the manufacturing hardware and extensible to three dimensions. New possibilities in terms of the design flexibility are foreseen by incorporating the concept of Wang tiles [24-27]. As opposed to commonly produced disordered structures, e.g., metal foams, the behavior of the jigsaw puzzle system augmented with Wang tiling would be fully predictable and customizable with respect to specific needs. After translating the concept to practical applications, automated assembly of tiles/modules based on optimized plans is envisaged.

\section{Acknowledgment}

The support by the Technology Agency of the Czech Republic and Ministry of Industry and Trade under research projects No. TACR TH02020420 and MPO FV10202, respectively, is gratefully acknowledged. In addition, this work was partly sponsored by CTU grant

No. SGS18/037/OHK1/1T/11.

\section{References}

[1] M. Granke, A. Gourrier, F. Rupin, K. Raum, F. Peyrin, M. Burghammer, A. Saded, P. Laugier, Microfibril orientation dominates the microelastic properties of human bone tissue at the lamellar length scale, PLoS ONE 8 (2013) e58043. doi:10.1371/journal.pone. 0058043.

[2] M. Wegener, Metamaterials beyond optics, Science 342 (2013) 939-940. doi:10.1126/ science.1246545.

[3] Z. Wu, R. Harne, K. Wang, Exploring a modular adaptive metastructure concept inspired by muscle's cross-bridge, Journal of Intelligent Material Systems and Structures 27 (2015) 1189-1202. doi:10.1177/1045389x15586451. 
[4] C. Schumacher, B. Bickel, J. Rys, S. Marschner, C. Daraio, M. Gross, Microstructures to control elasticity in 3d printing, ACM Transactions on Graphics 34 (2015) 136:1-136:13. doi:10.1145/2766926.

[5] D. Mousanezhad, S. Kamrava, A. Vaziri, Origami-based building blocks for modular construction of foldable structures, Scientific Reports 7 (2017) 14792. doi:10.1038/ s41598-017-13654-z.

[6] K.C. Cheung, N. Gershenfeld, Reversibly Assembled Cellular Composite Materials., Science 341, (2013) 1219-1221. doi.org/10.1126/science.1240889.

[7] M. Taylor, L. Francescony, M. Gerendás, A. Shanian, C. Carson, K. Bertoldi, Low porosity metallic periodic structures with negative Poisson's ratio, Materials 26 (2014) 2365-2370. doi:10.1002/adma.201304464.

[8] V. Nežerka, M. Somr, T. Janda, M. Doškář, J. Zeman, J. Novák, An Additively Manufactured Modular Metamaterial Composed of a Single Cell., Key Engineering Materials 722, (2017) 325-330. doi.org/10.4028/www.scientific.net/KEM.722.325.

[9] J. T. B. Overvelde, S. Shan, K. Bertoldi, Compaction through buckling in 2D periodic, soft and porous structures: Effect of pore shape, Advanced Materials 24 (2012) 2337-2342. doi : 10.1002 / adma. 201104395.

[10] J. T. B. Overvelde, K. Bertoldi, Relating pore shape to the non-linear response of periodic elastomeric structures, Journal of the Mechanics and Physics of Solids 64 (2014) 351-366. doi:10.1016/j.jmps.2013.11.014.

[11] R. Lakes, Foam structures with a negative Poisson's ratio, Science 235 (1987) 1038-1040. doi:10.1126/science.235.4792.1038.

[12] L. Ai, X.-L. Gao, Three-dimensional metamaterials with a negative poisson's ratio and a nonpositive coefficient of thermal expansion, International Journal of Mechanical Sciences 135 (2018) 101-113. doi:10.1016/j.ijmecsci.2017.10.042.

[13] I.A. Malik, M. Mirkhalaf, F. Barthelat, Bio-inspired "jigsaw"-like interlocking sutures: Modeling, optimization, 3D printing and testing., Journal of the Mechanics and Physics of Solids 102, (2017) 224-238. doi.org/10.1016/j.jmps.2017.03.003.

[14] C. Coulais, E. Teomy, K. de Reus, Y. Shokef, M. van Hecke, Combinatorial design of textured mechanical metamaterials, Nature 535 (2016) 529-532. doi : 10.1038 / nature18960.

[15] W. H. Peters, W. F. Ranson, Digital imaging techniques in experimental stress analysis, Optical Engineering 21 (1982) 427-431. doi:10.1117/12.7972925.

[16] J. Blaber, B. Adair, A. Antouniou, Ncorr: Open-source 2d digital image correlation matlab software, Experimental Mechanics 55 (2015) 1105-1122. doi:10.1007/ s11340-015-0009-1. 
[17] B. Florijn, C. Coulais, M. van Hecke, Programmable mechanical metamaterials, Physical Review Letters 113 (2014) 175503. doi:10.1103/physrevlett.113.175503.

[18] C. Coulais, D. Sounas, A. Alù, Static non-reciprocity in mechanical metamaterials, Nature 542 (2017) 461-464. doi : 10.1038 / nature21044.

[19] X. Zheng, H. Lee, T. H. Weisgraber, M. Shusteff, J. DeOtte, E. B. Duoss, J. D. Kuntz, M. M. Biener, Q. Ge, J. A. Jackson, S. O. Kucheyev, N. X. Fang, C. M. Spadaccini, Ultralight, ultrastiff mechanical metamaterials, Science 344 (2014) 1373-1377. doi: $10.1126 /$ science.1252291.

[20] N. Yang, J. L. Silverberg, Decoupling local mechanics from large-scale structure in modular metamaterials, Proceedings of the National Academy of Sciences 114 (2017) 3590-3595. doi:10.1073/pnas.1620714114.

[21] S. Tammas-Williams, I. Todd, Design for additive manufacturing with site-specific properties in metals and alloys, Scripta Materialia 135 (2017) 105-110. doi:10.1016/j. scriptamat. 2016.10 .030 .

[22] J. Ma, J. Song, Y. Chen, An origami-inspired structure with graded stiffness, International Journal of Mechanical Sciences 136 (2018) 134-142. doi:10.1016/j.ijmecsci. 2017.12 .026$.

[23] B. M. Tymrak, M. Kreiger, J. M. Pearce, Mechanical properties of components fabricated with open-source 3-D printers under realistic environmental conditions, Materials \& Design 58 (2014) 242-246. doi:10.1016/j.matdes.2014.02.038.

[24] H. Wang, Proving theorems by pattern recognition - II, Bell System Technical Journal 40 (1961) 1-41. doi:10.1002/j.1538-7305.1961.tb03975.x.

[25] J. Novák, A. Kučerová, J. Zeman, Microstructural enrichment functions based on stochastic Wang tilings, Modelling and Simulation in Materials Science and Engineering 21 (2013) 025014. doi:10.1088/0965-0393/21/2/025014.

[26] M. Doškář, J. Novák, A jigsaw puzzle framework for homogenization of high porosity foams, Computers \& Structures 166 (2016) 33-41. doi:10.1016/j.compstruc.2016.01. 003.

[27] M. Doškář, J. Zeman, D. Jarušková, J. Novák, Wang tiling aided statistical determination of the Representative Volume Element size of random heterogeneous materials, European Journal of Mechanics - A/Solids 70 (2018) 280-295. doi : 10 . $1016 / j$. euromechsol. 2017.12 .002 . 